III. Frauenförderung 


\title{
Gender Mainstreaming, Frauenförderung und Rechtsentwicklung im Hochschulbereich Vom Machtverhältnis zum Rechtsverhältnis und Verfahren
}

\author{
Margot Gebhardt-Benischke
}

Frauen und Männer, die, neugierig geworden durch verheißungsvolle Botschaften von Gender Mainstreaming als ,Innovation in der Gleichstellungspolitik" ${ }^{\text {*1 }}$ oder als „Chance für den Dialog zwischen den Geschlechtern “2 oder die hören, dass in den Hochschulen die Entwicklung ,,von der Frauenförderung zu Gender Mainstreaming ${ }^{* 3}$ in vollem Gange sei, meinen, dass sie als moderne, innovationsbewusste Bürgerinnen und Bürger darüber Bescheid wissen sollten, was Gender Mainstreaming eigentlich sei, können sich von der Bundesregierung informieren lassen. In einer von ihr herausgegebenen Broschüre, die auch ins Netz gestellt wurde ${ }^{4}$, heißt es:

\begin{abstract}
„Mit Kabinettsbeschluss vom 23. Juni 1999 hat die Bundesregierung die Gleichstellung von Frauen und Männern als durchgängiges Leitprinzip ihres Handelns anerkannt und beschlossen, diese Aufgabe mittels der Strategie des Gender Mainstreaming zu fördern. In der Gemeinsamen Geschäftsordnung der Bundesministerien ist dementsprechend die Verpflichtung aller Ressorts festgelegt, den Mainstream-Ansatz bei allen politischen, normgebenden und verwaltenden Maßnahmen der Bundesregierung zu beachten ( $\$ 2$ GGO). Hintergrund ist die rechtliche Verpflichtung der EU-Mitgliedstaaten aus dem Amsterdamer Vertrag. ${ }^{5}$ Art. 2 und 3 Abs. 2 EG-Vertrag verpflichten alle Mitgliedsländer zu einer aktiven und integrierten Gleichstellungspolitik im Sinne des Gender Mainstreaming. Auch Art. 3 Abs. 2 unseres Grundgesetzes stellt eine wichtige Rechtsgrundlage für eine aktive Gleichstellungspolitik dar. Der Staat muss sich aktiv um die ,tatsächliche Durchsetzung der Gleichberechtigung' und um die ,Beseitigung bestehender Nachteile' bemühen (Art. 3 Abs. 2 S. 2 GG).“
\end{abstract}

Auf die den neugierigen Bürgerinnen und Bürgern unterstellte Frage: „Gender Mainstreaming, was ist denn das?", gibt die Broschüre die Antwort, dass Gender Mainstreaming bedeute, bei allen gesellschaftlichen Vorhaben die unterschiedlichen Lebenssituationen von Frauen und Männern von vornherein und regelmäßig zu berücksichtigen, da es keine geschlechtsneutrale Wirklichkeit gebe. Mit der folgenden Inhalts-

1 Vgl. Bothfeld, Silke u. a. (Hrsg.): Gender Mainstreaming - Eine Innovation in der Gleichstellungspolitik, Frankfurt. 2002.

2 Vgl. Jansen, Mechthild M. u. a. (Hrsg.): Gender Mainstreaming - Herausforderung für den Dialog der Geschlechter, München: 2003.

3 Vgl. Kamphans, Marion: Von der Frauenförderung zum Gender Mainstreaming. In: Roloff, Christine/Selent, Petra (Hrsg.): Hochschulreform und Gender Mainstreaming - Geschlechtergerechtigkeit als Querschnittsaufgabe, Bielefeld: 2003, S. $165 \mathrm{ff}$.

4 Vgl. unter http://www.gender-mainstreaming.net (3. 3. 2004).

5 BGBl 1998, S. $387 \mathrm{ff}$. 
angabe will die Bundesregierung dazu anregen, einen ,,animierten Flash-Film zur spielerischen Erläuterung des Gender-Mainstreaming-Prinzips" herunterzuladen:

„Verschiedene Vogelarten (Storch, Schwan, Rabe, Pinguin, Specht, Pfau) versammeln sich vor dem Vogelrathaus und demonstrieren für neue Nester. Die Vogelverwaltung lässt daraufhin eine moderne Vogelsiedlung bauen. Beim Einzug in die neuen Nester müssen fast alle Vögel feststellen, dass ihre unterschiedlichen Lebenssituationen nicht berücksichtigt wurden. Die Störche passen nicht in das viel zu kleine Vogelhaus, der großen Schwalbenfamilie fehlt der Platz für die zahlreichen Kinder, den Pinguinen ist das Vogelhaus zu heiß und die Schwäne haben keinen Zugang zum Wasser. Die Bauverwaltung erfährt von Gender Mainstreaming und baut noch einmal neue Unterkünfte unter Berücksichtigung der verschiedenen Lebenssituationen der Vögel. Die Störche erhalten ein großes weiches Nest auf einem Baumstumpf, die Pinguine können in ein Iglu ziehen, die Schwalben erhalten ein Nest für die gesamte Familie und die Schwäne wohnen direkt am Wasser.“

Es ist übrigens wirklich lohnenswert, sich der angebotenen Diashow zu unterziehen. Die wohnungssuchenden Vögel tauchen nur als Pärchen auf, und das Gender-Mainstreaming-Handbuch, das dem verdutzten Chef des Vogelrathauses überreicht wird, enthält eine Definition von Gender Mainstreaming, die völlig neu ist: „Gender Mainstreaming bedeutet, bei allen gesellschaftlichen Vorhaben die unterschiedlichen Lebenssituationen der Beteiligten [sic!] von vornherein und regelmäßig zu berücksichtigen."

Auch wenn Vergleiche bekanntlich immer hinken, so lassen sie doch gewisse Schlussfolgerungen darauf zu, was die Vergleichende/den Vergleichenden dazu bewogen haben mag, gerade dieses und kein anderes Beispiel zum Vergleich heranzuziehen. Von dem gewählten Beispiel führt aber auch gar kein Weg zum Thema „Gleichberechtigung von Frauen und Männern“ oder in frappanter Weise vielleicht doch: Männer und Frauen werden wie Vögel-Männchen und Vögel-Weibchen als Pärchen/Familie gesehen und gedacht. In dieser gedachten Symbiose verschwinden alle Geschlechtergegensätze. Sie werden einfach weggedacht. Frauenförderung und Frauenpolitik ade? Das könnte doch die Lösung sein!

Die Abgrenzung von Gender Mainstreaming zu Frauenförderung bzw. die Bestimmung des Verhältnisses, in dem sie zueinander stehen, ist ein schwieriges Unterfangen. Das liegt weniger an dem Begriff der Frauenförderung als am Begriff des Gender Mainstreaming, der sich, von seiner häufig beklagten und entschuldigten „Unübersetzbarkeit" oder seiner ,Sperrigkeit" einmal völlig abgesehen, nur schwer fassen/erfassen, nur schwer greifen/begreifen lässt. Die Abgrenzung beider Bereiche ist jedoch notwendig. Während der Begriff der Frauenförderung im Laufe von mehr als drei Jahrzehnten relativ klare Konturen erhalten hat und durch gesetzliche Regelungen strukturiert und verankert wurde, ist das bei Gender Mainstreaming nicht der Fall.

Wenn sich das Gender-Mainstreaming-Prinzip, wie Kamphans es in ihrem Aufsatz „Von der Frauenförderung zum Gender Mainstreaming“ formuliert, „mit rasanter Ge- 
schwindigkeit auf die Hochschulen zubewegt ${ }^{* 6}$, könnte angesichts des Bildes vom rasenden Gender Mainstream schon eine gewisse Bangigkeit entstehen, dass er erhebliche Zerstörungen hinterlassen könnte - eine Bangigkeit, die nicht ganz grundlos ist. Einige Äußerungen geben durchaus dazu Anlass: Während früher, so ist zu lesen, Chancengleichheitsforderungen von den frauenpolitischen Aktivistinnen erhoben worden seien, werde heute die Forderung nach der Gleichstellung von Frauen und Männern auf höchster politischer Ebene verankert. Inzwischen sei aus der Förderung von Frauen die Förderung von Frauen und Männern gemäß dem Gender-Mainstreaming-Prinzip geworden. ${ }^{7}$ Manchen feministischen Frauenforscherinnen und -kämpferinnen würde gerade an dieser Stelle die Sache nicht schmecken, da deutlich werde, dass es bei der Realisierung von Gender Mainstreaming nicht primär um die notwendige Umsetzung von Frauenrechten gehe, sondern vor allem um die Verwirklichung zukunftsträchtiger Arrangements. Die Frage sei jedoch, ob solche „Zweckbündnisse“ nicht viel größere Realisierungschancen hätten als die früheren Ansätze der Frauenförderpolitik, die vor allem moralisch-ethisch begründet gewesen seien. ${ }^{8}$ Zahlreiche Autorinnen, die über Gender Mainstreaming schreiben, werfen interessante rhetorische Fragen auf. So stellt Stiegler z. B. die Fragen, ob Frauenförderung nun überholt und ob eine eigenständige Frauenpolitik noch erforderlich sei oder ob Gender Mainstreaming die Frauenpolitik lähmen könne, um dann zu antworten, dass das natürlich nicht der Fall sei. ${ }^{9}$ Oder es wird z. B. gefragt, ob die Frauenbeauftragten durch Gender Mainstreaming zum Auslaufmodell würden. ${ }^{10}$

\section{Das Geschlechterverhältnis, der Mainstream und die Frauenförderung}

Wenn Befürworterinnen des Gender-Mainstreaming-Prinzips die „traditionelle“ Frauenförderung gegen diesen „modernen“ Ansatz der Gleichstellungspolitik ausspielen, weil Frauenförderung einseitig sei und die Frauen als defizitär begreife, während Gender Mainstreaming gerade ihre Stärken zeigen wolle, ${ }^{11}$ ist ein kurzer Rückblick auf Ursa-

6 Vgl. Kamphans, Marion: Von der Frauenförderung zu Gender Mainstreaming. In: Roloff, Christine/Selent, Petra (Hrsg.): Hochschulreform und Gender Mainstreaming - Geschlechtergerechtigkeit als Querschnittsaufgabe, Bielefeld 2003.

7 Vgl. Ehrhardt, Angelika: Gender Mainstreaming - wo es herkommt, was es will und wie es geht. In: Jansen, Mechthild M./Röming, Angelika/Rohde, Marianne (Hrsg.): Gender Mainstreaming - Herausforderung für den Dialog der Geschlechter, München 2003, S. 13.

8 A. a. O., S. 27 f.

9 Vgl. Stiegler, Barbara: 10 Fragen und Antworten zum Konzept Gender Mainstreaming. http://www.landesfrauenrat-sachsen.de/gendermain/gender5.html (3. 3. 2004).

10 Vgl. Lischetti, Barbara: Die Frauenbeauftragte - ein Auslaufmodell? Erste Erfahrungen mit Gender Mainstreaming an der Universität Bern. In: Roloff, Christine/Selent, Petra (Hrsg.): Hochschulreform und Gender Mainstreaming - Geschlechtergerechtigkeit als Querschnittsaufgabe. Bielefeld: 2003, S. $182 \mathrm{ff}$.

11 Vgl. Stiegler, a. a. O. 
chen, Entstehungszusammenhänge und Ziele der „traditionellen“ Frauenförderung und Frauenpolitik angebracht.

Als am 1. 7. 1977 das Eherecht der BRD von Grund auf reformiert wurde und der Gesetzgeber das patriarchale Eheleitbild beseitigte, hatte damit der Mainstream eine radikale Veränderung des Geschlechterverhältnisses durchgesetzt. Mit der Aufgabe des Leitbilds der Hausfrauenehe, dem nunmehr für beide Ehepartner gegebenen Recht, erwerbstätig zu sein und der freien Scheidbarkeit der Ehe war auch die Verpflichtung zur Zahlung von nachehelichem Unterhalt geändert worden. Es gilt nunmehr der Grundsatz, dass nach einer Scheidung jede(r) für sich selbst zu sorgen hat, eine Veränderung, deren Radikalität, wie Schwab schreibt, der Öffentlichkeit erst nach und nach bewusst wurde. ${ }^{12}$ Wie sich aus der amtlichen Begründung ergibt, sah der Gesetzgeber einen natürlichen Zusammenhang zwischen der Aufgabe des Leitbilds der Hausfrauenehe und der Erwerbstätigkeit beider Ehepartner. Nachdem die Familienarbeit nicht mehr durch Gesetz der Ehefrau zugeschrieben war, war es nach Ansicht des Gesetzgebers weder notwendig noch gerechtfertigt, das Recht, erwerbstätig zu sein, für Mann und Frau unterschiedlich zu regeln. Das Recht, erwerbstätig zu sein, müsse der Frau schon deshalb ausdrücklich im Gesetz gegeben sein, ,weil eine eigene Erwerbstätigkeit der Ehefrau zu Selbständigkeit und Selbstbewusstsein verhilft (...) und ihr diese Möglichkeit vom Gesetzgeber nicht versagt werden darf" ${ }^{13}$ Allerdings hatte es der Mainstream abgelehnt, zugleich mit der Aufhebung des Leitbilds der Hausfrauenehe ein neues Leitbild an die Stelle zu setzen, etwa das der berufstätigen Ehefrau. Wie aus der amtlichen Begründung ebenfalls zu entnehmen ist, erwartete der Gesetzgeber von der Ehefrau eine verstärkte Rücksichtnahme und Nachgiebigkeit, insbesondere wenn Kinder zu erziehen sind. ${ }^{14}$ Uwe Diederichsen hat dies 1977 wie folgt kommentiert: ${ }^{15}$

„So erfreulich nun ein derartiges Emanzipationspathos des Gesetzgebers anmutet, so ist doch zu befürchten, daß auch diese Befreiungstat einseitig auf Kosten der Frau geht. Im Grunde wird von ihr jetzt verlangt, den Haushalt und die Kinder zu versorgen und sich daneben noch ein zusätzliches Selbstbewußtsein durch Aufnahme einer Erwerbstätigkeit zu besorgen. Mit der Aufgabe des Leitbilds der Hausfrauenehe entstehen keine zusätzlichen, für die Aufnahme einer Erwerbstätigkeit zur Verfügung stehenden Zeitreserven; und Umfragen haben gezeigt, daß die meisten Ehemänner trotz einer Erwerbstätigkeit ihrer Ehefrauen kaum mehr Arbeit im Haushalt übernehmen. Selbst eine Teilzeitbeschäftigung bedeutet für die meisten Ehefrauen eine sozialstaatlich kaum vertretbare Belastung. Es wäre also angebracht gewesen, zunächst die ökonomischen und sozialpsychologischen Rahmenbedingungen zu ändern, ehe man sich an die Demontage einer in gewisser Weise auch mit Privilegien verbundenen Rollenverteilung macht."

12 Vgl. Schwab, Dieter: Gleichberechtigung und Familienrecht im 20. Jahrhundert. In: Gerhard, Ute (Hrsg.): Frauen in der Geschichte des Rechts, München 1977.

13 Vgl. BTDrucks 7/650, S. 98.

14 A. a. O

15 Vgl. Diederichsen, Uwe: Die allgemeinen Ehewirkungen nach dem 1. Eherechtsgesetz und Ehevereinbarungen. In: Neue Juristische Wochenschrift 1977, S. 217 (220). 
Bei der Reform des Eherechts war Ende der 1960er Jahre unter dem Eindruck der Vollbeschäftigung von einer prognostizierten wirtschaftlichen Entwicklung ausgegangen worden, die zu einem wachsenden Bedarf an weiblichen Arbeitskräften führen würde. Dies war bekanntlich die Zeit, in der wegen der großen Nachfrage nach Arbeitskräften massenhaft „Gastarbeiter“ ins Land geholt wurden. ${ }^{16} \mathrm{Zu}$ Beginn der Reformarbeiten war dabei allen Beteiligten klar, dass das angestrebte Leitbild der modernen Ehe, in der beide Ehegatten voll ausgebildet sind und im Erwerbsleben stehen, noch eine kühne Utopie war. Der Entwurf wurde in der Öffentlichkeit überwiegend mit Empörung aufgenommen. Von der intendierten Abschaffung der Ehe als Versorgungseinrichtung sahen sich Millionen Frauen, die ihre Lebensplanung am gesetzlichen Leitbild der Hausfrauenehe ausgerichtet hatten, in ihrer Existenz bedroht. Der Spiegel schrieb 1970, als der Reformentwurf vorlag, dass die Reform des Eherechts ausgehe von der „Frau des Jahres 2000, selbstbewusst, berufstätig, politisch emanzipiert, die Partnerin in der Ehe ist und nach einer Ehescheidung mühelos ihrer Selbstverpflichtung nachkommt, während in der Realität eher die Frau des Jahres 1900 anzutreffen sei, wirtschaftlich abhängig und in der Ehe zuständig für Haushalt und Kinder. Wenn ein neues Eherecht noch im Jahre 2000 Bestand haben soll, muss es eine soziale Wirklichkeit antizipieren, einen weiblichen Bewußtseinsstand und einen Grad an Emanzipation, die erst noch zu schaffen sind. “17

Es schien nur eine Frage der Zeit, wann diese Voraussetzungen geschaffen sein würden. Die Disproportionalität zwischen der Wirklichkeit und den Gesetzesintentionen wurde erklärt mit dem Hinterherhinken der Wirklichkeit. Sie schien jedoch auflösbar durch einen vom Gesetzgeber selbst hervorgebrachten Reformzwang, wobei als Essentials zur Schaffung der inneren Gerechtigkeit des neuen Scheidungsrechts umfangreiche Reformen notwendig erschienen, die insbesondere die Beseitigung ungleicher Chancen im Erwerbsleben, Beseitigung der ungleichen Entlohnung von Männer- und Frauenarbeit, Beseitigung von Bildungs- und Ausbildungsbarrieren in einem neuen Bildungssystem, Ausbau von öffentlicher Kinderbetreuung, Ganztagsunterricht zur Entlastung der Mütter und zur besseren Ausbildung der Kinder und Vorschulerziehung zum Gegenstand haben sollten. ${ }^{18}$

Mit dem neuen Ehescheidungsrecht ist also das rechtliche Verhältnis der Geschlechter revolutioniert worden. Die Ehe, richtiger gesagt die patriarchale Ehe, in der die Frau auf die Hausfrauenrolle festgelegt war, war beseitigt. Damit war auch die Arbeit in Ehe und Familie als Existenzgrundlage beseitigt, weil jede Frau, auch wenn sie noch so treu gedient hat, die Kündigung bekommen kann und dann auf sich selbst gestellt ist. Wenn es früher hieß, die heiratet ja doch, die braucht keine lange Ausbildung, oder wenn für Mädchen familiennahe, kurze Ausbildungen empfohlen wurden, dann stand dahinter

16 Bis 1964 gab es bereits eine Million ausländischer ArbeitnehmerInnen in der BRD. Ihre Zahl stieg auf zwei Millionen im Jahr 1969 und erreichte ihren Höchststand 1973 mit 2,4 Millionen. Mit dem Beginn der Wirtschaftskrise im Herbst 1973 wurde die Anwerbung gestoppt und es gab erste Pläne für die Rückführung in die Heimatländer.

17 Der Spiegel 49/70, S. 82.

18 Vgl. Menschik, Jutta: Gleichberechtigung oder Emanzipation - Die Frau im Erwerbsleben der Bundesrepublik, Frankfurt 1976, S. 92 ff. 
das Ziel, den unkündbaren Beruf der Hausfrau und Mutter ergreifen zu wollen bzw. zu sollen. Zugleich, und das ist die Kehrseite der Medaille, sah sich jeder Mann auf Grund der vom Recht vorausgesetzten patriarchalen Ernährerrolle zumindest theoretisch lebenslangen Unterhaltsverpflichtungen ausgesetzt. Vor dem Hintergrund des alten patriarchalen Eherechts mit vorgegebener Rollenverteilung lassen sich in gewisser Weise sogar Regelungen ,moralisch legitimieren“, die alle Frauen, waren sie verheiratet oder nicht, wollten sie heiraten oder nicht, in Ausbildung und Beruf schlechter stellten als die Männer, waren sie doch alle potenzielle Hausfrauen und Mütter bzw. Ehemänner und Familienernährer. Bei der vorgegebenen Rollenverteilung war es verständlich, dass die eheinternen Aufgaben von Männern kaum übernommen wurden.

Mit der grundsätzlichen Befreiung der Frauen vom ehemännlichen Unterhalt, sprich mit der grundsätzlichen Verpflichtung, für sich selbst sorgen zu müssen, was Erwerbsarbeit bedeutet, müssen Frauen ebenso wie Männer die Möglichkeit haben, durch Erwerbsarbeit nachhaltig marktwirtschaftliches Einkommen und ein auch für das Alter existenzsicherndes Einkommen zu erzielen. Alles, was das behindert, muss beseitigt werden; faktische Nachteile, die typischerweise Frauen treffen, müssen durch begünstigende Regelungen ausgeglichen werden. Das ist keine „moralisch-ethische“ Frage, sondern die Quintessenz des Gleichberechtigungsgrundsatzes von Art. 3 Abs. 2 und 3 GG, wie er vom Bundesverfassungsgericht mehrfach, z. B. in der Entscheidung zur Verfassungswidrigkeit des Nachtarbeitsverbots für Arbeiterinnen bestätigt wurde. ${ }^{19}$

Das Fatale war und ist, dass das Leitbild der Hausfrau und Mutter bzw. der zuverdienenden Ehefrau noch lange nachwirkt bzw. durch den Mainstream propagiert und aufrechterhalten wurde und wird. Das Ehegattensplitting ist ein sinnfälliger Ausdruck dafür. Es gab damals bereits aussagekräftige Statistiken und Analysen über die weibliche Erwerbstätigkeit, über das Berufswahlverhalten von Frauen, ihre überwiegende Beschränkung auf typische Frauenberufe mit geringer Entlohnung und geringen Aufstiegschancen, die Altersarmut von Frauen, das Fehlen von Frauen in Führungsfunktionen in Wissenschaft, Wirtschaft, Verwaltung und Politik, das große Problem der Vereinbarkeit von Familie und Beruf, das Fehlen von Kinderkrippen, Kindergärten, Horten und Ganztagsschulen, die Tradierung der Hausfrauenrolle in den Schulbüchern oder die geringe Bereitschaft von Männern, Aufgaben in der Familie zu übernehmen. ${ }^{20}$

Gerade damals hätte es angesichts des radikalen Bruchs in der rechtlichen Regelung des Geschlechterverhältnisses nahe gelegen, dass die Reformen vom Mainstream in Angriff genommen wurden, um die ,von oben“ dekretierte formale Gleichberechtigung auch materiell zu vollenden, indem auf der Grundlage vorhandener Erkenntnisse die Rahmenbedingungen hätten verändert werden müssen. Tatsächlich hielt sich der Reformeifer in Grenzen. Die Wirtschaftskrise seit Herbst 1974 hatte zum massenhaften Abbau von Arbeitsplätzen geführt mit der Folge, dass viele Frauen, anders als bei der Reform des Eherechts vorausgesetzt, eben doch nicht ohne weiteres auf dem Arbeitsmarkt unterkommen konnten, sei es, dass die Arbeitsplätze fehlten, sei es, dass Arbeitsplätze eher an Männer vergeben wurden, sei es, dass Beschäftigungsverbote für Frauen

19 BVerfG, Urt. vom 28. 1, 1992 - BvR 1025/82, 1 BvL 16/83, 1BvL 10/91, NJW 1992, S. 964 ff.

20 Vgl. Menschik, Jutta: a. a. O. 
diesen den Zugang zu einzelnen Berufen verwehrten oder Kinderbetreuungseinrichtungen nicht geschaffen wurden. Auch die weit verbreitete Lohnungleichheit, häufig tarifvertraglich abgesichert, der Ausschluss von Frauen von Sonderzahlungen, die Benachteiligung von Teilzeitarbeitenden etwa beim beruflichen Aufstieg oder bei den Renten, hatten zur Folge, dass Frauen in ihrem grundgesetzlich garantierten Recht ebenso wie Männer existenzsicherndes Erwerbseinkommen zu erzielen, deutlich benachteiligt waren.

Nicht weil Frauen defizitäre Wesen sind oder weil es „moralisch-ethisch“ geboten ist, ihnen dieselben Entwicklungs- und Erwerbsmöglichkeiten zuzugestehen wie den Männern, sondern weil es eine ökonomische Notwendigkeit war, entstand die Forderung nach Beseitigung von Diskriminierung und nach Frauenförderung, nämlich in erster Linie nach Maßnahmen, die den Frauen den Anteil an Ausbildungs- und Arbeitsplätzen sichern sollten, die ihnen nach ihrer Qualifikation und ihrer Zahl zustehen, aber auf Grund diskriminierender Mechanismen, insbesondere wegen der nicht mehr zu rechtfertigenden Festlegung auf die Familienrolle verwehrt wurden.

Der Begriff der Frauenförderung hat seine juristische Definition zunächst in der vom Europäischen Parlament und dem Rat erlassenen „Richtlinie zur Verwirklichung des Grundsatzes der Gleichbehandlung von Männern und Frauen hinsichtlich des Zugangs zur Beschäftigung, zur Berufsbildung und zum beruflichen Aufstieg sowie in Bezug auf die Arbeitsbedingungen" - 76/207/EWG ${ }^{21}$-, deren Art. 4 Abs. 4 lautete:

„Diese Richtlinie steht nicht den Maßnahmen zur Förderung der Chancengleichheit von Männern und Frauen, insbesondere durch Beseitigung der tatsächlich bestehenden Ungleichheiten, die die Chancen der Frauen (...) beeinträchtigen, entgegen.“

Im Vertrag von Amsterdam wird in Art. 2 und 3 in den breit gefächerten Aufgabenkatalogen der Gemeinschaft auch die Gleichstellung von Männern und Frauen als Ziel aufgenommen, welches in Art. 141 Abs. 4 u. a. wie folgt konkretisiert wird:

„Im Hinblick auf die effektive Gewährleistung der vollen Gleichstellung von Männern und Frauen im Arbeitsleben hindert der Grundsatz der Gleichberechtigung die Mitgliedstaaten nicht daran, zur Erleichterung der Berufstätigkeit des unterrepräsentierten Geschlechts oder zur Verminderung oder zum Ausgleich von Benachteiligungen in der beruflichen Laufbahn spezifische Vergünstigungen beizubehalten oder zu beschließen."

Im September 2002 wurde die o.g. Richtlinie 76/207/EWG novelliert ${ }^{22}$. Sie nimmt nunmehr in Art. 2 Ziff. 8 auf Art. 141 Abs. 4 des Vertrags von Amsterdam Bezug und weist in Ziff. 14 der einleitenden Begründung darauf hin, dass die Mitgliedstaaten in erster Linie eine Verbesserung der Situation der Frauen im Erwerbsleben anstreben sollten.

Das bekannteste und lange Zeit sehr umstrittene Instrument der Frauenförderung war und ist die Quotenregelung, die vom Europäischen Gerichtshof mehrfach - wenngleich

21 ABl L 39 v. 14. 2. 1976.

$22 \mathrm{ABl} \mathrm{L} \mathrm{269/15}$ vom 2. 10. 2002. 
mit gewissen Restriktionen - bestätigt wurde. Quotenregelungen finden sich heute in den Gleichstellungsgesetzen der Länder, und auch das Gleichberechtigungs-/Gleichstellungsgesetz des Bundes enthält in $\S 2$ eine solche Regelung.

Der Begriff der Frauenförderung geht jedoch heute weit über die Quotenregelung hinaus. Insbesondere im Hochschul- und Wissenschaftsbetrieb hat der Begriff der Frauenförderung eine Diversifikation erfahren, die die vielfältigen Aufgaben, die Hochschulen haben, unter den Aspekten der Förderungsmöglichkeiten und -notwendigkeiten von Frauen aufgreift und differenzierte Handlungsansätze und Strategien entwickelt. Der Wissenschaftsrat, der auf zahlreiche Vorarbeiten von Frauenforscherinnen und Frauenpolitikerinnen zurückgreifen konnte, hat 1998 „Empfehlungen zur Chancengleichheit von Frauen in Wissenschaft und Forschung" veröffentlicht. ${ }^{23}$ Die Veröffentlichung ist gerade unter dem Eindruck des um sich greifenden Gender-Mainstreaming-Prinzips beachtlich, weil sich der Wissenschaftsrat unmissverständlich für Frauen fördernde Maßnahmen überall dort ausspricht, wo bekannte Brüche in der wissenschaftlichen Karriere von Frauen feststellbar sind und weil er im Besonderen die Empfehlung ausspricht, die Funktion der Frauenbeauftragten durch Personal- und Sachmittelausstattung zu stärken und das Instrument des Frauenförderplans einzusetzen.

\section{Das Geschlechterverhältnis oder vom Machtverhältnis zum Rechtsverhältnis und Verfahren am Beispiel Hochschulen}

Die Forderung nach Gleichheit, Gleichberechtigung und Chancengleichheit ist stets die Philosophie der Aufstrebenden. ${ }^{24}$ Mit jedem Schritt, der erreicht ist, ändert sich das Kräfteverhältnis, und es wird notwendig, Strategie und Taktik dem veränderten Kräfteverhältnis anzupassen. Die Entwicklung der Frauenfrage in den deutschen Hochschulen ist ein aufschlussreiches Beispiel dafür, wie sich das Kräfteverhältnis zwischen den männlich dominierten Hochschulen und ihren weiblichen Mitgliedern ständig verändert. Die Veränderung lässt sich als die Entwicklung vom Machtverhältnis zum Rechtsverhältnis bis hin zu einem durch Verfahren relativ geordneten Verhältnis beschreiben. Der Gender-Mainstreaming-Ansatz wird daran zu messen sein, ob er das Kräfteverhältnis in den Hochschulen zugunsten der Frauen verändert, ob er zumindest das Erreichte stabilisiert oder ob ihm das Potenzial innewohnt, das Erreichte zu gefährden.

Die Geschichte von Studentinnen und Wissenschaftlerinnen an deutschen Hochschulen ist eine noch junge. Erst 1908 fiel auch in Preußen das Verbot, dass Frauen sich an einer Hochschule immatrikulieren dürfen. In ihrem Aufsatz „Auf dem Weg in die Tempel der Wissenschaft. Zur Durchsetzung des Frauenstudiums in Deutschland" gibt v. Soden ${ }^{25}$ einen Überblick über den Kampf der Frauen um das Recht, studieren und

23 Wissenschaftsrat (Hrsg.). Empfehlungen zur Chancengleichheit von Frauen in Wissenschaft und Forschung 1998.

24 Vgl. Gusy, Werner: Der Gleichheitssatz. Neue Juristische Wochenschrift 1988, S. 2505 ff.

25 Vgl. v. Soden, Kristine in: Gerhard, Ute: Frauen in der Geschichte des Rechts, München: 1997, S. $617 \mathrm{ff}$. 
sich wissenschaftlich qualifizieren zu dürfen. Das Verbot der Habilitation, d. h. das Verbot, sich für eine Professur zu qualifizieren, fiel erst 1920. Mit den Mitteln des Rechts, mit Gesetzen, Verordnungen und Erlassen wurde den Frauen der Zugang zu den Universitäten verwehrt. Aus der Perspektive des Geschlechterdialogs gesehen bedeutet das: Solange Frauen nicht den Status von Mitgliedern der Gelehrtengemeinschaft erlangen konnten, konnte es keinen Dialog der Geschlechter innerhalb der Körperschaft Hochschule geben. Männer dialogisierten unter sich über Frauen, über ihr Wesen, ihre natürliche Bestimmung und ihre mangelnde Befähigung und Eignung zum Beruf der Wissenschaft.

Die Immatrikulation war der erste Schritt, die notwendige Vorbedingung dafür, dass sich in der Hochschule, in der Gelehrtenrepublik, ein Dialog zwischen Männern und Frauen überhaupt entwickeln konnte. Erst das Recht der Habilitation und die Berufung in eine Professur gab Frauen unter den Bedingungen der Ordinarienuniversität das abstrakte Recht zum Dialog innerhalb der Wissenschaftlergemeinschaft. Mit der Aufgabe der Ordinarienuniversität und dem Wechsel zur Gruppenuniversität war das Recht zum Dialog in der Hochschule nicht mehr nur an eine Professur geknüpft. Damit war zumindest die Möglichkeit gegeben, dass auch andere Gruppen sich in den Gremien zu Wort melden und mitreden durften. Das waren angesichts des niedrigen Anteils von Frauen an den Professuren, beim wissenschaftlichen Mittelbau und bei den Studierenden kaum Frauen. $^{26}$

Als Hochschulfrauen in den 1970er Jahren im Zuge der Neuen Frauenbewegung in außeruniversitären Zusammenhängen sich der Probleme bewusst wurden, dass die männliche Wissenschaft Frauen inhaltlich ausgrenzt und ihre zahlenmäßige Repräsen$\operatorname{tanz}$ an Hochschulen marginal war, formulierten sie ihre Forderungen an eine geschlechtergerechte Hochschule und konfrontierten damit die Hochschulen und die Öffentlichkeit. ${ }^{27}$ Insbesondere der „Arbeitskreis Wissenschaftlerinnen in NordrheinWestfalen“ formulierte bereits 1981 ein Memorandum zur Situation der Frauen im Wissenschaftsbetrieb und erarbeitete ein Antidiskriminierungsprogramm für den Wissenschaftsbetrieb, das grundlegend die weitere Entwicklung in den Hochschulen beeinflusst hat. ${ }^{28}$ Die Öffentlichkeit, mit der „ungeheuerlichen“ Behauptung konfrontiert, dass Frauen an den Hochschulen massiv diskriminiert würden, reagierte mit ungläubigem Staunen bzw. mit interessierter Skepsis. Ministerialbürokratie, Abgeordnete und Medien, von den konkreten Zahlen irritiert, erwarteten, dass die Behauptung der Dis-

26 Vgl. dazu die ausfiihrliche Situationsbeschreibung und -analyse von Schmidt-Harzbach, Ingrid: Kampf ums Frauenstudium - Studentinnen und Dozentinnen an deutschen Hochschulen. In: Frauen und Wissenschaft. Beiträge zur Berliner Sommeruniversität für Frauen. Berlin: 1977.

27 Vgl. dazu: Schlüter, Anne: Zehn Jahre Frauenpolitik im Wissenschaftsbetrieb - Ziele, Strategien, Ergebnisse. In: Gebhardt-Benischke, Margot/Stahr, Ingeborg (Hrsg.): Frauenpolitik im Wissenschaftsbetrieb: Perspektiven und Handlungsmöglichkeiten im Hochschul- und Wissenschaftsalltag, Alsbach/Bergstr. 1991.

28 Vgl. Knapp, Ulla: Anti-Diskriminierungsprogramm im Wissenschaftsbetrieb - zur Verankerung von Frauenforderungen in den Grundordnungen der Hochschulen am Beispiel der Universität-GH Wuppertal. In: Gebhardt-Benischke, Margot/Knapp, Ulla (Hrsg.): Was Frauen tun können, um die Hochschule zu verändern - Antidiskriminierungsprogramm im Wissenschaftsbetrieb, Alsbach/Bergstr.: 1984. 
kriminierung mit spektakulären Einzelfällen bewiesen werde. In den Hochschulen wurden die Ergebnisse schlicht ignoriert, wenn überhaupt einzelne Frauen das Recht erhielten, im Senat das Thema einzubringen. ${ }^{29}$ Wird diese historische Ausgangssituation einer juristischen Analyse unterzogen, so zeigt sich, dass sie drei juristische Charakteristika aufweist:

- Eines der juristischen Charakteristika bestand darin, dass „Frauen“ juristisch, d. h. als mögliche Subjekte und Beteiligte eines Verfahrens nicht existent waren. $\mathrm{Ob}$ und in welcher Weise sie von den Repräsentanten der Wissenschaft Gehör fanden oder sich zu Gehör brachten, war zunächst eine Frage des Wohlwollens, die allerdings zunehmend der Erkenntnis Platz machen musste, dass es politisch zweckmäßig sein könnte, Frauen die Gelegenheit zu geben, ihre Interessen zu artikulieren. Da Frauen als Gruppe jedenfalls verfahrensrechtlich nicht vorkamen, war es ohne Rechtsverstoß möglich, in den männerdominierten Hochschulen auf der Grundlage der ungeschriebenen patriarchalen Geschäftsordnung stillschweigend „Nichtbefassung" zu beschließen, wenn es um frauenrelevante Fragestellungen ging. Unter Nichtbefassung waren dabei alle bekannten und typischen Reaktionsweisen vertreten, vom Schweigen bis hin zu überheblichen, herablassenden und abwegigen Äußerungen zur Sache, also alle Formen der Un-Kommunikation.

- Ein weiteres Charakteristikum dieser Ausgangssituation war es, dass die Frauen, die sich außerhalb und ohne die Gewährleistung verfahrensrechtlicher Sicherungen zu Wort meldeten und die Hochschulen von deren Akteuren wie selbstverständlich in ein bestimmtes Verhältnis zueinander gestellt wurden, das der Rollenverteilung im Zivilprozess gleicht: Die Frauen wurden wie die klagende Partei in einem Zivilprozess mit dem Beweis dafür belastet, dass ihre Behauptungen wahr seien. Die Hochschulen hingegen, die „Beklagten", verlegten sich auf einfaches Bestreiten. Selbstherrlich und wie selbstverständlich wurde davon ausgegangen, dass bis zum Beweis des Gegenteils zu vermuten sei, dass die Hochschulen ihre Aufgaben diskriminierungsfrei und im Sinne des Gleichberechtigungsgebots wahrnehmen.

- Das dritte juristische Charakteristikum bestand darin, dass ein Diskriminierungsbegriff Anwendung fand, der nur die offene, direkte Benachteiligung erfasst. Dass dieser Diskriminierungsbegriff nicht nur im Alltagsverständnis verbreitet war, sondern zum damaligen Zeitpunkt auch in rechtswissenschaftlichen Fachkreisen noch ausgeprägt vertreten wurde, zeigt z. B. die Sachverständigenanhörung zum Prüfauftrag der Bundesregierung zum Thema „Kann die Situation der Frauen durch ein Antidiskriminierungsgesetz verbessert werden?“, die Anfang 1982 in Bonn durchgeführt wurde. Ein er-

Vgl. Schlüter: a. a. O. 
heblicher Teil der Sachverständigen vertrat dort die Ansicht, dass Benachteiligungen von Frauen nur einzelfallbezogen festgestellt werden können. ${ }^{30}$

Die Frauen, wiewohl juristisch inexistent, so doch real außerordentlich präsent, griffen zunächst den engen Diskriminierungsbegriff an, indem sie von Anfang an konkrete Zahlen ins Spiel brachten, um sichtbar zu machen, wie gering der Frauenanteil insbesondere an den exponierten Stellen im Wissenschaftsbetrieb ist. Unter dem Eindruck der Zahlen, für die es angesichts des Anteils der Studentinnen und der qualifizierten Examina von Frauen keine rationale Erklärung gab, vollzog sich allmählich ein Wandel in der Fremdeinschätzung der Hochschulen. Zugleich vollzog sich damit eine grundlegende Veränderung der quasi-zivilprozessualen Rollen- und Beweislastverteilung. Die Vermutung, dass die autonome Aufgabenwahrnehmung in den Hochschulen geschlechtergerecht erfolgt, geriet ins Wanken. Das entscheidende Indiz, dass sich in der Beurteilung der Hochschulen ein Wandel vollzogen hatte, war die Einführung des $\S 2$ Abs. 2 des Hochschulrahmengesetzes im Jahre 1985 und den entsprechenden Vorschriften in den Hochschulgesetzen der Länder mit der Verpflichtung, dass die Hochschulen bei der Wahrnehmung ihrer Aufgaben auf die Beseitigung der für Wissenschaftlerinnen bestehenden Nachteile hinzuwirken haben. Diese Vorschriften waren der sinnfällige Ausdruck dafür, dass nach Auffassung des Bundesgesetzgebers und der Gesetzgeber der Länder und damit der repräsentierten Allgemeinheit in den Hochschulen Nachteile für Frauen bestehen und dass die Hochschulen es bisher daran haben fehlen lassen, diese Benachteiligungen abzubauen und Frauen im Rahmen ihrer Aufgabe zur Förderung des wissenschaftlichen Nachwuchses gleichberechtigt zu fördern.

Parallel zu dieser Entwicklung vollzog sich in der Rechtswissenschaft ein Prozess der Veränderung bzw. Erweiterung des Diskriminierungsbegriffs, dessen Spezifikum darin besteht, dass unter Verzicht auf den Beweis der Diskriminierung im konkreten Fall eine Diskriminierung in der Form der strukturellen Diskriminierung dann angenommen wird, wenn eine statistisch signifikante Unterrepräsentanz von Frauen an einer bestimmten Berufs-, Funktions- oder Statusgruppe festzustellen ist und diese Unterschiede nicht anders als mit dem Geschlecht oder den Geschlechterrollen erklärt werden können. Diese zunächst anhand des privaten Arbeitsrechts entwickelte Diskriminierungstheorie ${ }^{31}$ wurde insbesondere von Benda in seiner gutachtlichen Stellungnahme für die Leitstelle Gleichstellung der Frau der Freien und Hansestadt Hamburg für den öffentlichen Dienst übernommen und weiterentwickelt. Nach Benda ist für den Bereich des öffentlichen Dienstes davon auszugehen, dass sich zahlenmäßige Diskrepanzen von Frauen als Folge einer Vielzahl von letztlich in den Strukturen des öffentlichen Dienstes als Institution begründeten Faktoren ergeben, weshalb er den Begriff der strukturellen Diskriminierung verwendet. $\mathrm{Ob}$ eine solche Diskriminierung vorliegt, soll nicht mehr

30 Vgl. dazu Gebhardt-Benischke, Margot (Hrsg.): Zur politischen und juristischen Diskussion eines Antidiskriminierungsgesetzes. In: Gebhardt-Benischke, Margot/Knapp, Ulla: Was Frauen tun können, um die Hochschule zu verändern - Antidiskriminierungsprogramm im Wissenschaftsbetrieb. Alsbach/Bergstr.: 1984, S. 202 ff.

31 Vgl. dazu Pfarr, Heide/Bertelsmann, Klaus: Gleichbehandlungsgesetz - zum Verbot der unmittelbaren und mittelbaren Diskriminierung von Frauen im Erwerbsleben, S. $107 \mathrm{ff}$. 
von dem empirischen Beweis des jeweiligen - zudem oft im Psychologischen - liegenden Diskriminierungsvorgangs abhängen. Zur Feststellung der strukturellen Diskriminierung genügt die Feststellung einer statistischen Diskrepanz. ${ }^{32}$ So, wie die Unterrepräsentanz eines Geschlechts in der Beschäftigtenstruktur eines Unternehmens dergestalt rechtliche Bedeutung erlangen kann, dass die Tatsache glaubhaft gemacht und die Vermutung begründet wird ( $\$ 611$ a Abs. 3 BGB), dass die Angehörigen des unterrepräsentierten Geschlechts in diesem Unternehmen diskriminiert werden, sind die Hochschulen aus der vormals unangefochtenen Position der Vermutung rechtmäßigen Handelns verdrängt und in eine Position gestellt worden, in der vermutet wird, dass sie die durch den Gleichberechtigungsgrundsatz und das Diskriminierungsverbot des Art. 3 Abs. 2 und 3 GG ,verpönte“ Einseitigkeit zulasten der weiblichen Mitglieder noch nicht aus ihrer Aufgabenwahrnehmung ,,verbannt“ haben.

Mit der Einführung der Funktion der Frauenbeauftragten an den Hochschulen seit Ende der 1980er Jahre hatte in der Körperschaft Hochschule, wenngleich noch rudimentär, der Übergang vom Geschlechterverhältnis als Machtverhältnis zum Geschlechterverhältnis als Rechtsverhältnis begonnen. Mit der allmählichen Durchsetzung von Beteiligungsrechten, die zunächst nur die Mitwirkung bei Einstellungen betrafen, die jedoch mittlerweile alle Aufgabenbereiche und Gremien einschließlich der leistungsorientierten Mittelvergabe betreffen - das Ergebnis kontinuierlicher Lobby-Arbeit der mittlerweile auf Bundes- und Landesebene zusammengeschlossenen Frauenbeauftragten und des politischen Drucks, den sie erzeugen konnten - hat auch das Verfahren, in dem der Geschlechterdialog in den Hochschulen stattfinden kann, eine Form gefunden.

Dass es mittlerweile in den Hochschulen ein gesetzlich geregeltes Verfahren der Kommunikation zur Frauenfrage gibt, dass die Frauenbeauftragte/Gleichstellungsbeauftragte weitgehende Mitwirkungsrechte hat, ist keineswegs die Gewähr dafür, dass sie mit allen Vorschlägen oder Beanstandungen auf Erfolgskurs ist. Je aufgeschlossener die Hochschulleitung oder die Fachbereichsleitungen sind, je besser die Frauenbeauftragte in ihrer Hochschule verankert ist, je kompetenter sie ihren Aufgabenbereich kommuniziert, desto erfolgreicher wird sie den Geist oder das Profil der Hochschule im Sinne einer geschlechtergerechten Hochschule beeinflussen und mitsteuern können.

\section{Gender Mainstreaming in Hochschulen: Fortschritt, Stillstand oder Rückschritt?}

Wenn von Gender Mainstreaming die Rede ist, fällt zunächst auf - so auch in der einleitenden Erklärung des Begriffs durch die Bundesregierung ${ }^{33}$, dass nicht auf das Grundgesetz der Bundesrepublik Deutschland rekurriert wird, sondern Art. 2 und 3 des Vertrags von Amsterdam bemüht werden, um die Verpflichtung zur Förderung der Gleich-

32 Vgl. Benda, Ernst: Notwendigkeit und Möglichkeit positiver Aktionen zugunsten von Frauen im öffentlichen Dienst. Gutachten im Auftrag der Leitstelle Gleichstellung der Frau der Freien und Hansestadt Hamburg 1985.

33 Siehe Fn. 4. 
stellung von Frauen und Männern zu legitimieren und dass diese Verpflichtung noch der besonderen Anerkennung bedarf. Art. 3 Abs. 2 und 3 GG werden nur als zusätzliche Grundlage erwähnt. Durch das Grundgesetz werden der Staat und alle Träger mittelbarer Staatsgewalt, dazu gehören auch die staatlichen Hochschulen, verpflichtet, die garantierte Gleichberechtigung von Frauen und Männer durchzusetzen und auf die Beseitigung bestehender Nachteile hinzuwirken. Ob die Träger der Staatsgewalt das anerkennen oder nicht, tut der Geltung des Gleichberechtigungsgrundsatzes keinen Abbruch. Der Gleichberechtigungsgrundsatz gilt nicht, weil er von der Regierung oder wem auch immer persönlich anerkannt wird oder weil internationale Konferenzen auch die Gleichberechtigung der Geschlechter fordern, sondern weil er eines der Grundprinzipien der Gesellschaft der Bundesrepublik ist, die sie in ihrer Verfassung festgeschrieben hat.

Noch bedenklicher ist es jedoch, wenn zur Propagierung des Gender-MainstreamingPrinzips in der BRD oder gar in den Hochschulen die Aktionsplattform der 4. Weltfrauenkonferenz herangezogen wird. Bereits im ersten Satz des mehr als hundert Seiten umfassenden Dokuments wird klargestellt: "The Platform for Action is an agenda for women's empowerment“. Um dieses „empowerment“, in der deutschen Übersetzung „Machtgleichstellung“, zu erreichen, sollen alle Entwicklungshilfemaßnahmen unter dem Geschlechteraspekt betrachtet werden, um sicherzustellen, dass die Fördermaßnahmen tatsächlich zielgerichtet auch die Frauen erreichen. Wichterich kritisiert, „dass der Gender-Ansatz nicht hält, was er versprochen hat, nämlich das Geschlechterverhältnis als Macht- und Ungleichverhältnis in den Griff zu nehmen. Vielmehr ist beobachtbar, dass er als Versöhnungs- und Harmonisierungsangebot definiert und die Friedenspfeife mit den Männern geraucht wird. “34

Wenn sich Gender Mainstreaming als Strategie zur Durchsetzung der Gleichberechtigung der Geschlechter in der Bundesrepublik auf Dokumente und Resolutionen berufen muss, die aus dem Zusammenhang der Entwicklungsarbeit und Entwicklungshilfe entstanden sind und die das Ziel verfolgen sollen, die Frauenarmut in der Welt zu bekämpfen und für alle Frauen und Mädchen dieser Welt das Menschenrecht auf Leben, Freiheit und Ausbildung zu verwirklichen, statt konsequent die Umsetzung des Gleichberechtigungsgebots auf der Grundlage des Grundgesetzes und der einfachen Gesetze durchzusetzen, dann ist das wohl eher als Rückschritt denn als Fortschritt zu bewerten.

Gender Mainstreaming als Politikansatz erfährt aus feministischer Perspektive viel Kritik. ${ }^{35}$ Es ist höchst aufschlussreich, dass auch die Autorinnen, die den Ansatz grundsätzlich befürworten, darauf hinweisen, dass es sich um einen höchst voraussetzungs-

34 Vgl. Wichterich, Christa: Frauenpolitik. In: NRO Frauenforum (Hrsg.). Mit Gender in die Zukunft? Tagungsdokumentation einer Fachtagung zur Genderperspektive in der Entwicklungszusammenarbeit 14.-15. 5. 1997, Stuttgart, S. 45-47.

35 Vgl. z. B. Widerspruch - Beiträge zu sozialistischer Politik, Bd. 44 mit Beiträgen von Stella Jegher, Susanne Schunter-Kleemann, Mascha Madörin, Barbara Nohr, Katharina Pühl, Claudia Michel/Flavia Vattolo, Natalie Imboden, Therese Wüthrich, Susanne Kappeler, Frigga Haug, Andrea Maihofer, Patricia Purtschert, Tove Soiland und Claudia v. Werlhof, Zürich 2003; Schwerpunktheft 2/2001 zu Gender Mainstreaming des Forum Wissenschaft mit Beiträgen von Heike Weinbach, Pia Gries, Ruth Holm, Bettina Stötzer, Barbara Nohr, Susanne Schunter-Kleemann, Sandra Kotlenga, Sandra Smykalla, Annette Kuhn, Christine Thürmer-Rohr, Stefan Möbius und Claudia v. Braunmühl. 
vollen Weg handelt, der nur gelingen kann, wenn er im Sinne des Top-down-Ansatzes von der Spitze der Institution vertreten wird. Immer wird auch die Gefahr gesehen, dass, wenn alle ihre Arbeit ,gendern“, vorhandene Strukturen der Frauenförderung gefährdet sein können. ${ }^{36}$ Der Begriff des Gender Mainstreaming schiebt sich praktisch wie eine Milchglasscheibe zwischen die klaren rechtlichen Vorgaben und diejenigen, die sie sie qua Amt umzusetzen haben. Gender Mainstreaming hat aber noch einen anderen bedenklichen Effekt: Um dem Begriff überhaupt einen Handlungsrahmen zu sichern, muss im Aufgabenbereich der Frauenförderung und Frauenpolitik gewildert werden bzw. es muss der Aufgabenbereich der Frauenförderung und Frauenpolitik auf ein unpolitisches Minimum reduziert werden. Das zeigt die Gegenüberstellung der Aufgabenbereiche und Methoden von Frauenpolitik und Gender Mainstreaming, wie sie z. B. von der Bundesregierung verbreitet wird: Gender Mainstreaming ziele auf Rahmenbedingungen und Strukturen, analysiere den Ist-Zustand anhand von Daten und wolle den Aspekt der Chancengleichheit von Frauen und Männern in alle Politikbereiche und politische Maßnahmen auf allen Ebenen einbringen, betreffe Frauen und Männer bzw. definierte Teile von ihnen, suche auch einen kurzfristigen Ausgleich, lege jedoch seine Hauptperspektive auf die langfristige Beseitigung von Ungleichheitsstrukturen, betreffe alle AkteurInnen, die an der Gestaltung, Umsetzung und Evaluierung politischer Konzepte beteiligt seien.

Frauenpolitik hingegen, so meint die Bundesregierung, ziele direkt auf den Abbau von Benachteiligungen, reagiere auf Ungleichbehandlungen im politischen, wirtschaftlichen, gesellschaftlichen, sozialen und kulturellen Leben, betreffe die Zielgruppe der Frauen bzw. einen oder mehrere Teile der weiblichen Bevölkerung, suche schnelle Lösungen, die eine unmittelbare Verbesserung der Lebenssituation von Frauen nach sich ziehen, werde von eigenen, für Frauenpolitik zuständigen Organisationseinheiten, die darüber hinaus in andere Politikbereiche Vorschläge zur Umsetzung einbringen, umgesetzt.

Das muss nun vollends verwirren. War es vielleicht gar nicht Frauenpolitik, sondern Gender Mainstreaming, wenn Frauen fast dreißig Jahre lang die Hochschulen mit Statistiken traktiert haben, die patriarchalen Strukturen in den Hochschulen angegangen sind, die Dialogfähigkeit und ihre Mitwirkung an der Mittelverteilung in den Hochschulen durchgesetzt, ihre Beteiligung und Mitwirkungsrechte in allen Hochschulgremien erreicht, in langwierigen Aushandlungsprozessen die politischen Akteure auf allen Ebenen mobilisiert, Förderprogramme für Wissenschaftlerinnen durchgesetzt, feministische Wissenschaft salonfähig gemacht, wenn sie Kinderbetreuungseinrichtungen für studierende Eltern durchgesetzt oder Sommerschulen zur Erhöhung des Studentinnenanteils an ingenieur- und naturwissenschaftlichen Studiengängen initiiert haben und dergleichen mehr?

Die Frage, die sich stellt, ist nicht die, ob es nicht wirklich schön wäre, wenn sich plötzlich alle, die in der Hochschule Verantwortung tragen, weil es „,von oben“ so gewollt ist, diesen Aufgaben stellen und sie kreativ weiterentwickeln, nachdem sie ihr

36 Vgl. statt vieler Stiegler, Barbara: Wie Gender in den Mainstream kommt. In: Bothfeld, Silke u. a. (Hrsg.): Gender Mainstreaming - Eine Innovation in der Gleichstellungspolitik, Frankfurt: 2002, S. 19 (38 f.). 
Gender-Mainstreaming-Trainingsprogramm mit Zertifikat absolviert haben. Die Frage ist, ob die teuer eingekaufte Gender-Kompetenz nicht an anderer Stelle zum Sparen zwingt, nämlich bei dem „kostenintensiven“ Verfahren des Dialogs zwischen den Geschlechtern, wie es sich in den letzten drei Jahrzehnten langsam, aber konsequent entwickelt hat. Sollte es nicht wenigstens ein bisschen stutzig machen, dass gar keine Kritik am Gender-Mainstreaming-Prinzip aus der Ecke derjenigen kommt, die sich gegen jede konkrete gesetzliche Regelung zur Änderung des Geschlechtervertrages - um nur die Quotenregelung, die Abschaffung des Ehegattensplittings oder ein Antidiskriminierungsgesetz für die private Wirtschaft zu nennen - mit allen ihnen reichlich zu Gebote stehenden juristischen, politischen und publizistischen Mitteln und durchaus mit Erfolg zu wehren wussten und wissen?

Der Dialog der Geschlechter über den ständig neu zu verhandelnden Geschlechtervertrag ist unabdingbar notwendig. Solange allerdings als Essential für den Dialog der Geschlechter die Bereitschaft „der da oben“ oder anders ausgedrückt das Top-downPrinzip als unabdingbar gelten, steht und fällt der Dialog mit dieser Bereitschaft. 
